\title{
NLRP1 and NLRP3 polymorphisms in mesothelioma patients and asbestos exposed individuals a population-based autopsy study from North East Italy
}

\author{
Violetta Borelli ${ }^{1}$, Ronal R Moura ${ }^{2}$, Elisa Trevisan ${ }^{1}$ and Sergio Crovella ${ }^{2,3^{*}}$
}

\begin{abstract}
NRLP1 (rs12150220, rs9889625, rs9900356, rs6502867, rs2670660) and NLRP3 (rs35829419, rs10754558) polymorphisms have been analyzed in 69 subjects with documented asbestos exposure and death for malignant pleural mesothelioma and 59 patients with documented asbestos exposure but death for other causes, all from a North East Italy. No association was found between NLRP1 and NLRP3 polymorphisms and susceptibility to develop mesothelioma using the general, dominant or recessive models. Also haplotype analysis did not reveal any significant association with mesothelioma. Our findings, being controversial with respect to another study on Italian patients, do suggest the need of further studies to unravel the contribution of NLRP1 and NLRP3 in susceptibility to mesothelioma.
\end{abstract}

Keywords: Mesothelioma, Inflammasome, NLRP1 and NLRP3 polymorphisms, Asbestos exposure, Haplotypes

\section{Findings}

The involvement of inflammasome in susceptibility to develop malignant pleural mesothelioma (MPM), considering its capacity to sense asbestos fibres has been already reported [1] and several papers have been published so far on this topic [2-4].

Genetic susceptibility to mesothelioma has been widely analyzed and some recent genome wide studies pointed out several susceptibility genes (i.e. BAP1, NF2, CDKN2A, CUL1, SLC7A14, THRB, CEBP350, ADAMTS2, ETV1, $P V T 1$ and MMP14), but genetic variants in inflammasome genes such as NLRP3 and NLRP1 have not been associated to mesothelioma [5-7]. However, Girardelli et al. [8] reported an association of rs12150220 NLRP1 polymorphism with mesothelioma in Italian patients the presence of $\mathrm{T}$ allele conferred a major risk (OR 1.79) to acquire the disease.

\footnotetext{
* Correspondence: crovelser@gmail.com

${ }^{2}$ Department of Genetics, Federal University of Pernambuco, Recife, Brazil ${ }^{3}$ Department of Medical, Surgical and Health Sciences, University of Trieste, Trieste, Italy

Full list of author information is available at the end of the article
}

With the aim of replicating Girardelli et al. [8] results, we analyzed NRLP1 and NLRP3 polymorphisms in subjects exposed to asbestos from North East Italy.

We collected 128 subjects deceased during the period 1980-2000, of which 69 with documented asbestos exposure and death for MPM (AEM) and 59 patients with documented asbestos exposure but death for causes different from MPM and other asbestos related diseases (AENM), used as controls. All subjects were characterized based on information available in medical records and the MPM diagnosis was performed by histologic examination. For the AENM group objective signs confirming asbestos exposure were the presence of hyaline plaque at autopsy $\left(\geq 2^{\circ}\right)$ at pleural level, presence of asbestos bodies $(A B)$ in routine lung sections and (for most cases) counts of $A B$, expressed as number of $A B$ per gram of dry lung tissue, $\geq 10.000 / \mathrm{g}$.

Genomic DNA of AEM and AENM was extracted from formalin-fixed and paraffin-embedded archived autopsy heart tissue slices (about 40-50 slices with a thickness of 5-7 $\mu \mathrm{m}$ were cut from the same paraffin block) using the QIAsymphony DSP DNA mini kit (QIAGEN, Hilden, Germany) and eluted in 50ul of TE buffer.

\section{Biomed Central}

(c) 2015 Borelli et al. This is an Open Access article distributed under the terms of the Creative Commons Attribution License (http://creativecommons.org/licenses/by/4.0), which permits unrestricted use, distribution, and reproduction in any medium, provided the original work is properly credited. The Creative Commons Public Domain Dedication waiver (http:// creativecommons.org/publicdomain/zero/1.0/) applies to the data made available in this article, unless otherwise stated. 
Quantitative and qualitative evaluation of extracted DNA was performed using NanoDrop ND-1000 (Termo Scientific, Wilmington, USA); electrophoresis on agarose gel allowed us to verify the degree of DNA degradation.

We genotyped two NLRP3 polymorphisms (rs35829419, cSNP and rs10754558, Tag SNP) and five NLRP1 polymorphisms (rs12150220, cSNP; rs9889625, Tag SNP; rs9900 356, Tag SNP; rs6502867, Tag SNP; rs2670660, Tag SNP) using the following Taqman genotyping assays: NLRP3 C_25648615_10, C__26052028_10; NLRP1 C__1600653_10, C__1274455_10, C_29796119_10, C_29222211_20 and C__1600689_10. Allelic specific PCR reactions have been run on ABI 7500 real time instrument (Life Technologies, Foster City, CA). SDS software (Life Technologies) allowed allelic discrimination and genotyping.

Table 1 summarizes the distribution of NLRP1 and $N L R P 3$ polymorphisms genotype counts among the AEM and AENM groups (a graphic representation is also provided as Additional file 1: Figure S1). All markers were in Hardy-Weinberg Equilibrium (HWE) in both AEM and AENM groups with the exception of NLRP1 rs9900356 SNP in AEM group and NLRP3 rs35829419, which was not in HWE in AENM and AEM.

No association was found between NLRP1 and NLRP3 polymorphisms and susceptibility to develop mesothelioma using the general, dominant or recessive models. Moreover, we evaluated the formation of haplotypes in NLRP1 and NLRP3 genes. We detected two blocks for NLRP1: Haplo1 (rs9900356, rs6502867 and rs9889625; $\mathrm{D}^{\prime}=1$ ) and Haplo2 (rs12150220 and rs2670660; D' = 0.83). The two NLRP3 SNPs also formed a haplotype $\left(\mathrm{D}^{\prime}=1\right)$. We estimated the haplotype frequencies of the NLRP1 and NLRP3 polymorphisms in AEM and AENM individuals and no association with susceptibility to the disease was found; haplotype distributions are in Additional file 2: Table S1; Additional file 3: Figure S2, reports the haploblocks found for NLRP1 and NRLP3 SNPs.

So the association (for both rs12150220 SNP and rs12150220- rs2670660 haplotype) with susceptibility to mesothelioma reported by Girardelli et al. [8] has not been replicated in our study, also performed in Italian patients and controls (our samples were from Monfalcone, an industrial area with endemic exposition to asbestos).

However, in the study of Girardelli et al. NLRP1 and NRLP3 polymorphisms have been evaluated in patients with mesothelioma and controls living in a geographic area known to be at risk of asbestos exposure, while in our study both mesothelioma patients and controls had documented asbestos exposure (see Additional file 4: Table S2 for details), so our genetic analysis has been performed on more homogeneous cohorts.

Girardelli et al. [8] et al. hypothesized a role for NLRP1 inflammasome in the context of mesothelioma on the
Table 1 NLRP1 and NLRP3 polymorphisms genotype counts among the AEM and AENM groups

\begin{tabular}{|c|c|c|c|c|c|}
\hline \multicolumn{6}{|c|}{ NLRP1 } \\
\hline SNP & $\operatorname{AEM}(n=69)$ & AENM $(n=59)$ & OR & $95 \% \mathrm{Cl}$ & $\mathrm{p}$-value \\
\hline \multicolumn{6}{|c|}{ rs9900356 } \\
\hline$T / T$ & 40 & 34 & 1.00 & Ref & \\
\hline $\mathrm{T} / \mathrm{C}$ & 25 & 23 & 0.92 & $0.45-1.91$ & 0.9781 \\
\hline $\mathrm{C} / \mathrm{C}$ & 4 & 2 & 1.70 & $0.29-9.86$ & 0.8645 \\
\hline \multicolumn{6}{|c|}{ rs6502867 } \\
\hline $\mathrm{T} / \mathrm{T}$ & 36 & 34 & 1.00 & Ref & \\
\hline $\mathrm{T} / \mathrm{C}$ & 23 & 19 & 1.14 & $0.53-2.46$ & 0.8835 \\
\hline $\mathrm{C} / \mathrm{C}$ & 10 & 6 & 1.57 & $0.52-4.80$ & 0.6008 \\
\hline \multicolumn{6}{|c|}{ rs9889625 } \\
\hline $\mathrm{G} / \mathrm{G}$ & 29 & 25 & 1.00 & Ref & \\
\hline$A / G$ & 25 & 21 & 1.03 & $0.47-2.26$ & 0.8911 \\
\hline $\mathrm{A} / \mathrm{A}$ & 15 & 13 & 0.99 & $0.40-2.48$ & 0.8242 \\
\hline \multicolumn{6}{|c|}{ rs12150220 } \\
\hline $\mathrm{A} / \mathrm{A}$ & 20 & 18 & 1.00 & Ref & \\
\hline T/A & 29 & 25 & 1.04 & $0.45-2.40$ & 0.9118 \\
\hline $\mathrm{T} / \mathrm{T}$ & 20 & 16 & 1.13 & $0.45-2.81$ & 0.9849 \\
\hline \multicolumn{6}{|c|}{ rs2670660 } \\
\hline $\mathrm{A} / \mathrm{A}$ & 17 & 15 & 1.00 & Ref & \\
\hline$A / G$ & 34 & 30 & 1.00 & $0.43-2.34$ & 0.8283 \\
\hline $\mathrm{G} / \mathrm{G}$ & 18 & 14 & 1.13 & $0.42-3.04$ & 1.0000 \\
\hline \multicolumn{6}{|c|}{ NLRP3 } \\
\hline SNP & $\operatorname{AEM}(n=69)$ & AENM $(n=59)$ & OR & $95 \% \mathrm{Cl}$ & \\
\hline \multicolumn{6}{|c|}{ rs35829419 } \\
\hline $\mathrm{C} / \mathrm{C}$ & 60 & 52 & 1.00 & Ref & \\
\hline $\mathrm{A} / \mathrm{C}$ & 6 & 6 & 0.87 & $0.26-2.85$ & 0.9452 \\
\hline $\mathrm{A} / \mathrm{A}$ & 3 & 1 & 2.60 & $0.26-25.76$ & 0.7379 \\
\hline \multicolumn{6}{|c|}{ rs10754558 } \\
\hline$C / C$ & 23 & 23 & 1.00 & Ref & \\
\hline$C / G$ & 33 & 25 & 1.32 & $0.61-2.87$ & 0.6152 \\
\hline $\mathrm{G} / \mathrm{G}$ & 13 & 11 & 1.18 & $0.44-3.18$ & 0.9369 \\
\hline
\end{tabular}

OR Odds Ratio, $\mathrm{Cl}$ Confidence Interval, Ref reference genotype (the most frequent)

basis of NLRP1 lung expression (alveolar macrophages and epithelial cells), a location where this innate immune protein could act as sensor of asbestos fibres. The authors suggested a possible functional role for NLRP1 functional SNP rs12150220, indicating the presence of the $\mathrm{T}$ allele as able to imbalance NLRP1 production. Since no functional data are already available in the literature for rs 12150220 variation, we performed an in silico search with SIFT and Polyphen softwares able to predict an eventual detrimental effect of the SNP: both SIFT (0.16) and Polyphen (0.415) predictive values indicate no damaging effect for NLRP1 rs12150220 SNP. Being aware of the limitations of in silico 
SNPs functional prediction, the hypothesized role of this variation should be functionally verified.

In conclusion, our study, performed on patients and controls with homogeneous asbestos exposure, does not confirm the involvement of NLRP1 inflammasome in the susceptibility to mesothelioma in Italian patients, pointing out the necessity to analyze a wider number of individuals, such as those used in the most recent genome wide studies, to deeper investigate the contribution of the inflammasome to the multifactorial mesothelioma.

\section{Additional files}

Additional file 1: Figure S1. Histogram plot representing the distribution of NLRP1 and NLRP3 SNPS genotypes in subjects with documented asbestos exposure and death for pleural mesothelioma (AEM, dark bar) and individuals with documented asbestos exposure but death for other causes (AENM, grey bar). No statistically significant difference has been detected within AEM and AENM (see Table 1 in the main text of the article for $p$ values). (DOCX $660 \mathrm{~kb}$ )

Additional file 2: Table S1. Haplotype frequencies of the NLRP1 and NLRP3 polymorphisms in AEM and AENM individuals (DOCX 26 kb)

Additional file 3: Figure S2. Haploview representation of the haploblocks found for NLRP1 and NRLP3 SNPS (DOCX $40 \mathrm{~kb}$ )

Additional file 4: Table S2. Characteristics of selected malignant pleural mesothelioma (MPM) cases and controls in a Necropsy Series, Monfalcone Area 1980-2000. (DOCX 42 kb)

\section{Abbreviations}

AB: Asbestos bodies; MPM: Malignant pleural mesothelioma; AEM: Subjects with documented asbestos exposure and death for pleural mesothelioma; AENM: Individuals with documented asbestos exposure but death for other causes; HWE: Hardy-Weinberg Equilibrium.

\section{Competing interests}

The authors declare that they have no competing interests.

\section{Authors' contributions}

VB designed the study, performed the sample selection and contributed to manuscript writing. RRM performed samples genotyping and statistical analysis. ET participated in sample selection and DNA extraction and genotyping. SC extracted DNA and wrote the manuscript. All authors read and approved the final manuscript.

\section{Acknowledgements}

We would like to thank all the staff of the Monfalcone's Hospital Pathological Anatomy Unit for their valuable technical assistance and Dr. Claudio Bianchi for his precious help in data collection process.

\section{Funding}

This work was supported by grants funded by University of Trieste, FRA 2011 (Local Research Grant) and by Associazione Esposti Amianto di Monfalcone.

\section{Author details}

${ }^{1}$ Department of Life Sciences, University of Trieste, Trieste, Italy. ${ }^{2}$ Department of Genetics, Federal University of Pernambuco, Recife, Brazil. ${ }^{3}$ Department of Medical, Surgical and Health Sciences, University of Trieste, Trieste, Italy.

Received: 14 May 2015 Accepted: 20 July 2015

Published online: 01 August 2015

\section{References}

1. Dostert C, Pétrilli V, Van Bruggen R, Steele C, Mossman BT, Tschopp J. Innate immune activation through Nalp3 inflammasome sensing of asbestos and silica. Science. 2008;320:674.
2. Hillegass JM, Miller JM, MacPherson MB, Westbom CM, Sayan M, Thompson $\mathrm{JK}$, et al. Asbestos and erionite prime and activate the NLRP3 inflammasome that stimulates autocrine cytokine release in human mesothelial cells. Part Fibre Toxicol. 2013;10:39.

3. Mossman BT, Shukla A, Heintz NH, Verschraegen CF, Thomas A, Hassan R. New insights into understanding the mechanisms, pathogenesis, and management of malignant mesotheliomas. Am J Pathol. 2013;182:1065.

4. Carbone $\mathrm{M}$, Yang $\mathrm{H}$. Molecular pathways: targeting mechanisms of asbestos and erionite carcinogenesis in mesothelioma. Clin Cancer Res. 2012;18:598.

5. Guo G, Chmielecki J, Goparaju C, Heguy A, Dolgalev I, Carbone M, et al. Whole-exome sequencing reveals frequent genetic alterations in BAP1, NF2, CDKN2A, and CUL1 in malignant pleural mesothelioma. Cancer Res. 2015;75:264.

6. Panagopoulos I, Gorunova L, Davidson B, Heim S. Novel TNS3-MAP3K3 and ZFPM2-ELF5 fusion genes identified by RNA sequencing in multicystic mesothelioma with $\mathrm{t}(7 ; 17)(\mathrm{p} 12 ; \mathrm{q} 23)$ and $\mathrm{t}(8 ; 11)(\mathrm{q} 23 ; \mathrm{p} 13)$. Cancer Lett. 2015;357:502.

7. Matullo G, Guarrera S, Betti M, Fiorito G, Ferrante D, Voglino F, et al. Genetic variants associated with increased risk of malignant pleural mesothelioma: a genome-wide association study. PLoS One. 2013;8(4):e61253.

8. Girardelli M, Maestri I, Rinaldi RR, Tognon M, Boldorini R, Bovenzi M, et al. NLRP1 polymorphisms in patients with asbestos-associated mesothelioma. Infect Agent Cancer. 2012;7:25.

\section{Submit your next manuscript to BioMed Central and take full advantage of:}

- Convenient online submission

- Thorough peer review

- No space constraints or color figure charges

- Immediate publication on acceptance

- Inclusion in PubMed, CAS, Scopus and Google Scholar

- Research which is freely available for redistribution 